\title{
Recurrent appendicitis after appendectomy
}

\author{
Kathryn L. Martin MD, Michele Weir MD, Peter B. Engbers MD, Brian Taylor MD
}
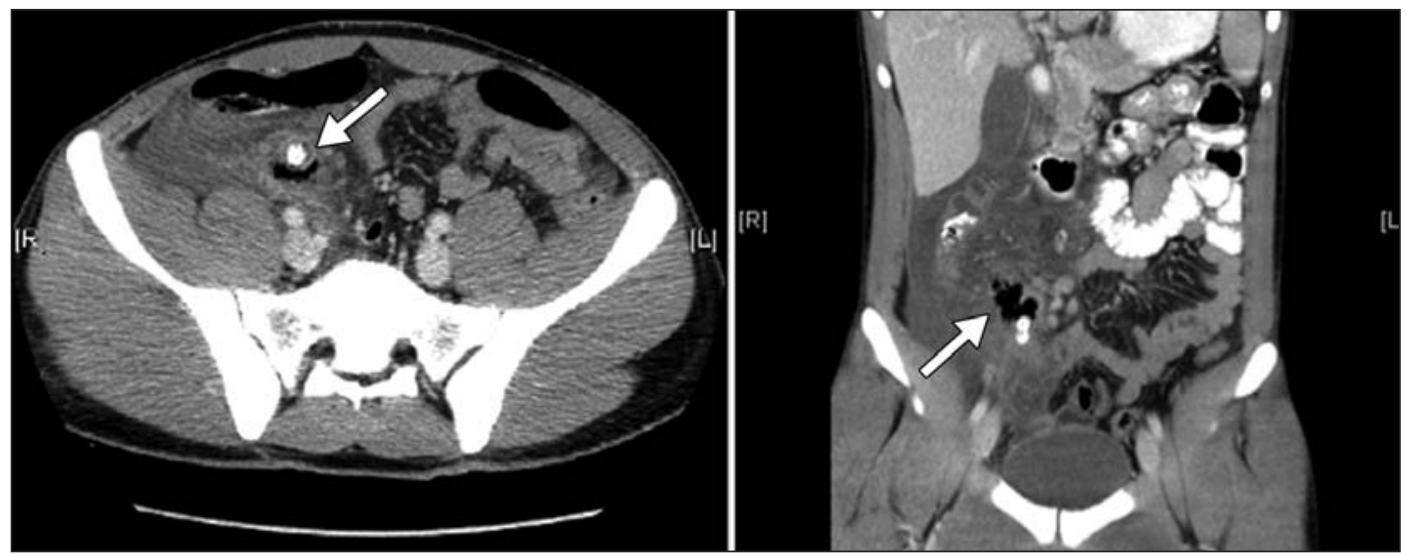

Figure 1: Computed tomographic images in a 19-year-old man with a history of laparoscopic appendectomy for perforated appendicitis, showing extraluminal gas, a complex fluid collection and fecaliths (arrows) in the right paracolic gutter and pelvis, consistent with complicated appendicitis. No appendix was identified.

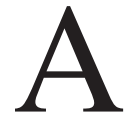

19-year-old man with a history of laparoscopic appendectomy for perforated appendicitis presented with a five-day history of vague periumbilical pain that had localized to the right lower quadrant. Ultrasonography was nonspecific, and the patient was admitted with a diagnosis of gastroenteritis. His clinical condition deteriorated overnight as fever, tachycardia and peritonitis developed. Laboratory investigations showed leukocytosis $\left(24\right.$ [normal 4-10] $\left.\times 10^{9} / \mathrm{L}\right)$. Computed tomography (CT) showed free fluid, extraluminal gas and fecaliths in the right paracolic gutter and pelvis (Figure 1). The diagnosis of complicated appendicitis was suggested.

The patient was taken to the operating room, where mobilization of the cecum showed a large abscess containing a perforated and necrotic retrocecal appendix. The site of the previous appendectomy was not identified. Final pathology showed a suppurative appendix with perforation. A review of the previous appendectomy specimen showed a gangrenous appendix, leading to the current diagnosis of appendiceal duplication with metachronous appendicitis.

Retrocecal duplication of the appendix, as seen in our patient, is thought to result from the persistence of the transient cecal protuberance at the sixth embryonic week. ${ }^{1}$ Duplication of the appendix is estimated to occur in $0.004 \%-$ $0.009 \%$ of the population. ${ }^{1}$ Duplication of the appendix may be detected intraoperatively or on ultrasonography, barium enema or $\mathrm{CT}^{2}{ }^{2}$

The most common cause of right lower quadrant pain, fever and leukocytosis is appendicitis. If a patient presenting with this clinical scenario has a history of appendectomy, the possibilities of stump appendicitis and recurrent appendicitis secondary to appendiceal duplication should be considered..$^{23}$ Although both conditions are rare (the exact incidences are unknown $n^{2.3}$ ), physicians should be aware of this possibility so as not to assume that previous appendectomy precludes recurrent appendicitis. Failure to recognize this possibility may lead to delays in treatment and may result in complications such as perforation, abscess formation and sepsis.

\section{References}

1. Cave AJ. Appendix vermiformis duplex. J Anat 1936;70:283-92.

2. Griffiths EA, Jagadeesam J, Fasih T, et al. Bifid vermiform appendix: a case report. Curr Surg 2006;63:176-8.

3. Liang MK, Lo HG, Marks JL. Stump appendicitis: a comprehensive review of literature. Am Surg 2006;72:162-6.
Competing interests: None declared.

This article has been peer reviewed.

Affiliations: From the Division of General Surgery (Martin, Taylor), and the Department of Pathology (Weir), London Health Sciences Centre, London, Ont.; and the Department of Pathology (Engbers), Woodstock General Hospital, Woodstock, Ont.

Correspondence to: Dr. Kathryn Martin, kmartin2008@meds.uwo.ca

CMAJ 2012. DOI:10.1503 /cmaj.110018 